\title{
DROGAS ANTIFÚNGICAS PARA PEQUENOS E GRANDES ANIMAIS
}

\author{
ANTIFUNGICAL DRUGS FOR SMALL AND LARGE ANIMALS
}

\author{
Márcia de Oliveira Nobre ${ }^{1}$ Patrícia da Silva Nascente $^{2}$ \\ Mario Carlos Meireles ${ }^{3}$ Laerte Ferreiro ${ }^{4}$
}

\section{- REVISÃO BIBLIOGRÁFICA -}

\section{RESUMO}

As drogas antifúngicas são classificadas em agentes químicos clássicos e atuais e em antibióticos. Os agentes químicos clássicos são aqueles medicamentos que apresentam de maneira geral espectro de ação reduzido contra fungos, atuando como fungistático de modo indireto ao modificar as condições locais, como por exemplo o iodo, os ácidos graxos e derivados, ácido salicílico, tolnaftato e tolciclato. Os agentes químicos atuais são representados pelos imidazóis e triazóis, flucitosina e alilaminas e os antibióticos representados pelos poliênicos (anfotericina $B$, nistatina e natamicina) e a griseofulvina. A descrição de cada antifúngico considerado será apresentada no trabalho.

Palavras-chave: antifúngicos, azóis, triazois, alilaminas, anfotericina $B$, griseofulvina.

\section{SUMMARY}

The antifungal drugs are classified in classic and current chemical agents and in antibiotics. The chemists classic agents are those medicines that present in a general way reduced action spectrum against fungi, having a fungistatic effect in an indirect way when modifying the local conditions, as for instance the iodine, acids fat and derived, acid salicilic, tolnaftate and tolciclate. The current chemical agents are represented by imidazole and triazole, flucytosine and allylamines and the antibiotics represented by the polyene (amphotericin B, nystatin and natamycin) and the griseofulvin. The discretion of each antifungal drug will be presented in the work.
Key words: antifungical, azole, triazole, allylamine, amphotericin B, griseofulvin.

\section{INTRODUÇÃO}

A lista de substâncias químicas com ação antifúngica é bastante extensa, mas ainda muito restrita ao ser comparada com o número de drogas antibacterianas disponíveis. Como conseqüência das infecções por fungos representarem o parasitismo de um organismo eucariótico sobre um outro eucariótico (homem e animal), com diferenças fisiológicas muito pequenas, quando comparado a infecções bacterianas, é necessário que as drogas antifúngicas tenham aplicação clínica adequada, com o mínimo de efeitos colaterais importantes (LACAZ \& NEGRO, 1991; ALVES et al., 1999). As drogas antifúngicas exercem ações fungistáticas ou fungicidas, direta ou indiretamente. Os antifúngicos têm características especiais quanto ao mecanismo de ação, via de administração, ação em micoses superficiais e, ou sistêmicas, podendo ser classificados com base no sítio-alvo e estrutura química, sendo que estes atuam em sua maioria na membrana celular (azóis, anfotericina e nistatina), excetuando-se a fluocitosina e a griseofulvina, que

${ }^{1}$ Médico Veterinário, Mestre, Faculdade de Veterinária, Universidade Federal de Pelotas (UFPEL).

${ }^{2}$ Médico Veterinário bolsista de Mestrado da CAPES, Pós-graduação em Veterinária, UFPEL

${ }^{3}$ Médico Veterinário, Doutor, Professor do Departamento de Veterinária Preventiva, Faculdade de Veterinária, UFPEL, Campus Universitário, 96010-900, Pelotas, RS, Brasil. Email:meireles@ufpel.tche.br . Autor para correspondência.

${ }^{4}$ Médico Veterinário, Doutor, Professor do Setor de Micologia, Departamento de Patologia Clínica, Faculdade de Veterinária, Universidade Federal do Rio Grande do Sul. 
atuam na síntese do ácido nucleico (LACAZ \& NEGRO, 1991). Nos últimos 10 anos, o uso de novas e mais efetivas drogas (antibacterianas e imunossupressoras) aumentaram a sobrevida dos pacientes, porém tornaram estes organismos mais suscetíveis às infecções micóticas (RICHARDSON \& WARNOCK, 1993; ALVES, et al., 1999; POLAK, 1999).

Fundamentado no crescente
conhecimento e no relato de casos de infecções fúngicas superficiais, subcutâneas ou profundas, em animais, somado ao pouco número de medicamentos veterinários com princípios antifúngicos, desenvolvemos esta revisão bibliográfica com o objetivo de descrever as principais drogas antifúngicas, suas recomendações (Tabela 1 e 2) e seus efeitos em pequenos e grandes animais.

Tabela 1 - Indicações dos antifúngicos mais freqüentemente utilizados nas micoses animais e daqueles em fase de pesquisa.

\begin{tabular}{|c|c|}
\hline ANTIFÚNGICOS & MICOSES \\
\hline Captan & Dermatofitose \\
\hline $\begin{array}{l}\text { Solução de iodo, } \\
\text { tolnaftato e tolciclato }\end{array}$ & Dermatomicoses \\
\hline Iodeto de potássio & Esporotricose \\
\hline Clotrimazol & $\begin{array}{l}\text { Otites fúngicas, dermatofitose, malassezioses, candidoses e } \\
\text { aspergilose nasal }\end{array}$ \\
\hline Econazol & Dermatofitose, malassezioses e candidoses \\
\hline Miconazol & $\begin{array}{l}\text { Dermatofitose, malassezioses, candidoses, mastite fúngica } \\
\text { bovina, queratomicoses de eqüinos }\end{array}$ \\
\hline Enilconazol & $\begin{array}{l}\text { Aspergilose aviária, aspergilose nasal de caninos e eqüinos, } \\
\text { dermatofitose e malasseziose }\end{array}$ \\
\hline Cetoconazol & Micoses superficiais, subcutâneas e sistêmicas \\
\hline Itraconazol & Micoses superficiais subcutâneas e sistêmicas \\
\hline Fluconazol & Micoses superficiais, subcutâneas e sistêmicas \\
\hline Voriconazol* & $\begin{array}{l}\text { Micoses sistêmicas, principalmente na candidose, aspergilose e } \\
\text { criptococose em pacientes debilitados, imunossuprimidos ou } \\
\text { quando houve resistência para outro antifúngico }\end{array}$ \\
\hline Posoconazol* & $\begin{array}{l}\text { Micoses sistêmicas, principalmente na aspergilose, em } \\
\text { pacientes debilitados, imunossuprimidos ou em falha de outro } \\
\text { antifúngico }\end{array}$ \\
\hline Terbinafina & Dermatofitose \\
\hline Flucitosina & Criptococose \\
\hline Anfotericina B & Micoses sistêmicas \\
\hline Nistatina & $\begin{array}{l}\text { Micoses superficiais, otites e metrites, principalmente } \\
\text { candidose }\end{array}$ \\
\hline Nistatina Lipossomal & $\begin{array}{l}\text { Micoses sistêmicas, principalmente na candidose, criptococose } \\
\text { e aspergilose em pacientes debilitados, imunossuprimidos ou } \\
\text { quando houve resistência para outro antifúngico }\end{array}$ \\
\hline Natamicina & Mastites fúngicas bovinas e queratomicose de eqüinos \\
\hline Griseofulvina & Dermatofitose \\
\hline $\begin{array}{l}\text { Pneumocandinas e } \\
\text { Echinocandinas * }\end{array}$ & $\begin{array}{l}\text { Micoses sistêmicas, principalmente em candidose e aspergilose } \\
\text { em pacientes debilitados, imunossuprimidos ou quando houve } \\
\text { resistência para outro antifúngico }\end{array}$ \\
\hline
\end{tabular}

*Antifúngicos em fase I, II ou III de estudo para liberação comercial do medicamento

\section{AGENTES QUÍMICOS}

No grupo dos agentes químicos estão incluídos aqueles medicamentos que apresentam, de maneira geral, espectro reduzido contra fungos, e outros que não são antimicóticos no sentido direto, mas atuam como fungistático de modo indireto ao modificar as condições locais. Entre esses últimos, estão sais de metais pesados (mercúrio, prata, zinco, cobre); substâncias contendo íons oxidantes (permanganato de potássio, água oxigenada e cromatos); derivados do anel benzênico (ungüento de whitfield); sais quaternários de amônio; ácidos graxos orgânicos e derivados (ácido propiônico, undecilênico, butírico, láctico e caprílico); violeta genciana utilizada em solução aquosa ou alcoólica a $1 \%$; tintura de castellani elaborada a base de fuccina e a cloroiodohidroxiquinoleína ou clioquinol; haloprogin; tolnaftato e tolciclato; compostos sulfurados, halógenos ou halogenóforos e derivados (iodo, cloro, bromo) (LACAZ \& NEGRO, 1991; ARENAS, 1993). O iodo é empregado como tintura a $1 \%$ em solução alcoólica ou aquosa, sendo um fungicida de amplo espectro, econômico e eficaz, indicado principalmente em dermatofitoses, sendo contra indicado em pele inflamada, por ser irritante. $\mathrm{O}$ iodeto de potássio é o tratamento mais adequado para a esporotricose humana, na forma fixa e linfangite, mas não se conhece com exatidão o mecanismo de ação; ele não atua "ïn vitro" contra o Sporothrix schenckii, sendo que sua ação in vivo parece estar relacionada com o estímulo da fagocitose. As dosagens indicadas para cães são $44 \mathrm{mg} / \mathrm{kg}$ e para gatos $22 \mathrm{mg} / \mathrm{kg}$ a cada 8 ou 12 horas. Os felinos são bastante sensíveis as preparações de iodetos, devendo ser cuidadosamente monitorados em busca de evidências de iodismo como: febre, ptialismo, anorexia, hiperexcitabilidade, pelagem seca, vômito ou diarréia (WOLF \& GREGORY, 1992). Captan (N-triclorometil-mercapto-4ciclo-hexeno-1,2-dicarboxamina) é um antifúngico agrícola, utilizado para tratamento da dermatofitose, principalmente de bovinos e eqüinos, 
Tabela 2 - Indicação e dosagens dos antifúngicos para uso sistêmico nas micoses superficiais, subcutâneas e profundas de cães e gatos.

\begin{tabular}{lcccc}
\hline & \multicolumn{4}{c}{ MICOSE } \\
\cline { 2 - 5 } ANTIFÚNGICOS & $\begin{array}{c}\text { SUPERFÍCIAIS } \\
(\mathrm{mg} / \mathrm{kg} / \mathrm{dia})\end{array}$ & $\begin{array}{c}\text { SUBCUT ÂNEAS E } \\
\text { PROFUNDAS } \\
(\mathrm{mg} / \mathrm{kg} / \mathrm{dia})\end{array}$ \\
\hline & CÃES & GATOS & CÃES & GATOS \\
Cetoconazol & $5-30$ & $5-30$ & $10-30$ & $10-30$ \\
Itraconazol & até 5 & $1,5-3$ & $10-20$ & $10-20$ \\
Fluconazol & $2,5-5,5$ & $2,5-5$ & $2,5-5,5$ & $2,5-5$ \\
Terbinafina & - & Até 20* & - & - \\
Griseofulvina & $25-50$ & $25-50$ & - & - \\
Flucitosina & - & - & $125-250$ & $125-250$ \\
Anfotericina B & - & - & $0,2-0,8 * *$ & $0,1-0,8 * *$ \\
\hline
\end{tabular}

* a cada 24 ou 48 horas;

** deve ser avaliado a condição geral do animal para estipular o intervalo entre doses.

através de banhos de aspersão a 3\% (BLOOD \& RADOSTIS, 1991).

Os azóis são antimicóticos, químicos, de largo espectro, atuando sobre leveduras e bolores, apresentam um anel imidazol livre unido a outros anéis aromáticos por meio de uma união $\mathrm{N}-\mathrm{C}$ em posição 1. Os imidazóis foram descobertos em 1949, sendo que o nitrato de imidazol foi utilizado experimentalmente pela primeira vez em 1967. Atualmente dispõe-se de vários derivados do imidazol para uso sistêmico ou tópico no tratamento de diversas infeções micóticas (Tabela 1). A ação fungistátitica ou fungicida é dependente da concentração da droga. Recentemente os triazólicos têm recebido maior destaque, sobretudo o fluconazol e o itraconazol, ambos com largo espectro de ação e efeitos tóxicos bastante reduzidos. Pela sua freqüente utilização, tem-se observado resistência entre os fungos, principalmente por espécies de Candida (LACAZ \& NEGRO, 1991; ARENAS, 1993; ALVES, et al., 1999).

O principal mecanismo de ação dos azólicos é a inibição da biossíntese do ergosterol, que é importante para a integridade e a manutenção da função da membrana celular dos fungos. Os imidazóis inibem a incorporação do acetato de ergosterol, inibindo a lanosterol desmetilase, por interferência no citocromo P-450 da levedura, trazendo como consequiência alterações na fluidez $\mathrm{e}$ permeabilidade da membrana citoplasmática do fungo, prejudicando a captação dos nutrientes, o que se traduz por inibição do crescimento fúngico, originando alterações morfológicas que resultam em necrose celular (RICHARDSON \& WARNOCK,
1993; ALVES et al., 1999). A metabolização dos azóis é principalmente por via hepática, sendo os efeitos colaterais mais comuns náuseas e vômitos quando utilizados por via sistêmica, além de eritema, ardência, descamação, edema, prurido, urticária e formação de vesículas no uso tópico (SANDE \& MANDELL, 1987; ARENAS, 1993).

O clotrimazol é utilizado topicamente no tratamento de dermatofitose, candidose e malasseziose (SAWYER et al., 1975a), com absorção inferior a $0,5 \%$ após aplicação à pele intacta; na vagina a absorção oscila entre 3 e $10 \%$, permanecendo concentrações da droga por até três dias após a aplicação (SANDE \& MANDELL, 1987). O clotrimazol a $1 \%$ está indicado para cães no tratamento de otites externas com envolvimento de Malassezia pachydermatis (LOBELL et al., 1995) e em dermatofitoses por Trichophyton mentagrophytes e Microsporum canis (McCURDY et al., 1981) e aspergilose intranasal (MATHEWS $\boldsymbol{e t}$ al., 1998; SMITH et al., 1998), sendo neste último caso utilizado em forma de instilação, podendo como efeito colateral ocorrer edema de faringe e faringite (CAULKETT et al., 1997). O clotrimazol para grandes animais está indicado para eqüinos no tratamento de queratite micótica por Aspergillus e para bovinos no tratamento da mastite micótica, utilizando via intramamária solução ou pomadas com 100 a $200 \mathrm{mg} /$ dia no quarto afetado (WEIGHT \& AHLERS, 1982; PRESCOTT \& BAGGOT, 1991).

O econazol e o miconazol são imidazóis indicados para uso tópico devido a sua toxicidade. $\mathrm{O}$ econazol é considerado menos ativo contra as leveduras, mas o mais ativo para fungos filamentosos. O miconazol possuí uma estrutura química semelhante ao econazol, sendo indicado nas dermatofitose, malasseziose e nas candidoses mucocutânea, penetrando rapidamente na camada córnea da pele, persistindo por quatro dias após sua aplicação, sendo que menos de $1 \%$ é absorvido na circulação (SAWYER $\boldsymbol{e t}$ al., 1975b; HEEL $\boldsymbol{e t}$ al., 1978; LACAZ \& NEGRO, 1991; RICHARDSON \& WARNOCK, 1993). O miconazol em solução aquosa tem sido indicado para o tratamento intramamário em vacas com mastite por Candida $\mathrm{sp}$ (PRESCOTT \& BAGGOT, 1991) e na queratomicoses de eqüinos com envolvimento de Aspergillus sp e Fusarium sp (ANDREW et al., 1998) na dermatite seborréica dos cães associada a malasseziose através do uso de shampoos combinado com outros medicamentos (BOND et al., 1995). Outros imidazóis como tioconazol, oxiconazol, isoconazol, bifonazol, sulconazol são também utilizados topicamente (ARENAS, 1993). 
Estudos in vitro com enilconazol Microsporum canis (DAVIS \& LEGENDRE, 1994; WHITE-WEITHERS \& MEDLEAU, 1995; JAHAM et al., 2000), Microsporum gypseum, Trichophyton mentagrophytes, Trichophyton verrucosum, Malassezia pachydermatis e Aspergillus spp (JAHAM et al., 2000). O enilconazol, indicado somente para uso veterinário e com mínimos efeitos colaterais, tem tido sucesso no controle da aspergilose aviária e em desinfecção de aviários, utilizando suspensão do medicamento diluído em 1:1000; e no tratamento da dermatofitose canina, felina e eqüina e na malasseziose, o medicamento é utilizado na concentração final de 0,2\% (JAHAM $\boldsymbol{e t}$ al., 2000). Estudos recentes relatam o ótimo efeito do enilconazole no tratamento da aspergilose nasal em cães, através de instilação nasal com suspensão de enilconazole, à 5 ou 10\% em concentração final, durante 45-60 minutos ou utilizando dose total de $10 \mathrm{mg} / \mathrm{kg}$ duas vezes ao dia durante 7 a 10 dias (McCULLOUGH et al., 1998; BRAY et al., 1998; CLERCX, 2000) e no tratamento da aspergilose da bolsa gutural em eqüino (VAN NIEUWSTADT \& KALSBEEK, 1994).

O cetoconazol é indicado em uso tópico ou por via oral, tendo amplo potencial terapêutico para o tratamento de infecções micóticas superficiais e sistêmicas. A distribuição do cetoconazol é limitada e sua penetração no líquido cefaloraquidiano é mínima. A resposta ao tratamento oral é relativamente lenta, sendo menos útil em infecções graves e agudas. A atividade antimicótica do cetoconazol frente aos fungos Coccidioides immitis, Cryptococcus neoformans, Histoplasma capsulatum e Blastomyces dermatitidis é atingida em concentrações de $0,125 \mu \mathrm{g} / \mathrm{m} \ell$ até $0,5 \mu \mathrm{g} / \mathrm{m} \ell$. Os valores correspondentes para Sporothrix schenckii, Candida sp e Aspergillus sp variam entre $6 \mu \mathrm{g} / \mathrm{m} \ell$ até concentrações iguais ou maiores que $100 \mu \mathrm{g} / \mathrm{m} \ell$. (SANDE \& MANDELL, 1987; LACAZ \& NEGRO，1991; RICHARDSON \& WARNOCK, 1993).

Estudos in vitro sobre a atividade fungicida do cetoconazol frente a amostras de fungos filamentosos e leveduriformes isolados de animais têm sido variada. Concentrações a partir de $10 \mu \mathrm{g} / \mathrm{m} \ell$ têm ação fungicida sobre Trichophyton verrucossum e Malassezia pachydermatis, enquanto na fase leveduriforme do Sporothrix schenckii, no Cryptococcus neoformans, no Histoplasma capsulatum e no Blastomyces dermatitidis são necessários $20 \mu \mathrm{g} / \mathrm{m} \ell$ e para o Coccidioides immitis $50 \mu \mathrm{g} / \ell$. A concentração de $50 \mu \mathrm{g}$ de cetoconazol $/ \mathrm{m} \ell$ sobre Trichophyton mentagrophytes e Microsporum canis foi moderadamente fungicida. Independente das concentrações de cetoconazol testadas (10 a $1.000 \mu \mathrm{g} / \mathrm{m} \ell$ ), os melhores efeitos fungistático e fortemente fungistático foram obtidos respectivamente frente ao Trichophyton equinum e Microsporum nanum, enquanto para Aspergillus fumigatus, Candida albicans e Candida tropicalis a atividade do medicamento foi baixa (GABAL, 1986). A concentração inibitória mínima (CIM) média para amostras de Malassezia pachydermatis isoladas do meato acústico de cães foi de $0,019 \mu \mathrm{g} / \mathrm{m} \ell$ e $0,012 \mu \mathrm{g} / \mathrm{m} \ell$ para amostras isoladas da pele (COUTINHO, 1997). O cetoconazol apresentou a melhor atividade in vitro contra a $\boldsymbol{M}$. pachydermatis, quando comparado a outros antifúngicos (clotrimazol, miconazol, nistatina e pimaricina) com um CIM na concentração de $0,02 \mu \mathrm{g} / \mathrm{m} \ell$ atingindo 11 amostras incluindo uma cepa padrão de 42 amostras estudadas (UCHIDA $\boldsymbol{e t}$ al., 1990). Para M. pachydermatis a resistência in vitro do cetoconazol tem sido baixa, variando entre 0 e 6,7\% (UCHIDA et al., 1990; COUTINHO, 1997).

A dosagem recomendada para $\mathrm{O}$ tratamento das dermatofitoses em cães e gatos é de 5 a $10 \mathrm{mg} / \mathrm{kg}$ de peso/dia, durante quatro a seis semanas. A dosagem do cetoconazol para o tratamento das micoses sistêmicas de cães é de $10 \mathrm{mg} / \mathrm{kg}$ de peso, três vezes ao dia, ou $20 \mathrm{mg} / \mathrm{kg} / \mathrm{dia}$ em dose única ou fracionada; em gatos a dose é $20 \mathrm{mg} / \mathrm{kg}$ de peso, administrada duas vezes ao dia em dias alternados ou $10-15 \mathrm{mg} / \mathrm{kg}$ de peso administrados diariamente. Em todas as micoses sistêmicas, o tratamento com cetoconazol deve ser prolongado pelo menos durante quatro semanas após a cura clínica da enfermidade (NOXON et al.,1982; MORIELLO, 1986). JAHAM et al. (2000) sugerem dosagens de $5-30 \mathrm{mg}$ de cetoconazol $/ \mathrm{kg} / \mathrm{dia}$ para o tratamento de micoses caninas e felinas (Tabela 2). No cão, o cetoconazol atinge picos de concentrações séricas dentro de duas horas após uma dose padrão oral de $10 \mathrm{mg} / \mathrm{kg}$. A absorção máxima ocorre em ambiente ácido, sendo recomendado administrar no paciente em jejum; apresenta meia-vida de duas horas e sendo eliminado em média em dez horas. Cães que receberam quatro vezes a dose recomendada, isto é $40 \mathrm{mg} / \mathrm{kg} / \mathrm{dia}$ durante um ano, apresentaram poucos efeitos adversos, entretanto nas doses de 60 a $80 \mathrm{mg} / \mathrm{kg}$ observou-se a hepatoxicidade, através do aumento das enzimas hepáticas e colângio-hepatite (MORIELLO, 1986). Em outro estudo, os cães receberam 10 a $30 \mathrm{mg} / \mathrm{kg} / \mathrm{dia}$ e sofreram queda nas concentrações de cortisol em repouso e pós-ACTH (Hormônio 
adrenocorticotrópico), bem como queda na testosterona e aumento na progesterona, sem a presença de outros sinais clínicos (WILLARD et al., 1986), o que evidencia o cuidado que se deve ter no uso deste produto em animais reprodutores (ARONSON \& AUCOIN, 1992). Já os gatos apresentaram mais problemas gastrintestinais do que os cães, quando foram submetidos ao regime de $30 \mathrm{mg}$ de cetoconazol $/ \mathrm{kg} / \mathrm{dia}$, durante 30 dias, não apresentando mudanças hormonais significativas (ARONSON \& AUCOIN, 1992).

O itraconazol é um derivado triazólico sintético, que apresenta um largo espectro de ação nas micoses superfíciais (dermatofitose, candidose, malasseziose) e sistêmicas (candidose sistêmica, aspergilose, histoplasmose, esporotricose e cromomicose). A biodisponibilidade de itraconazol é máxima quando a ingestão se faz imediatamente após uma refeição e a eliminação é bifásica, com uma meia-vida terminal em um dia. Os níveis da droga nos tecidos queratinizados, especialmente na pele, são cinco vezes superiores aos níveis plasmáticos (ARENAS, 1993; RICHARDSON \& WARNOCK, 1993). O itraconazol testado in vitro frente a 30 amostras de Malassezia pachydermatis coletadas do meato acústico e pele de cães demonstrou um CIM médio de $0,004 \mu \mathrm{g} / \mathrm{m} \ell$, com $73,3 \%$ de amostras sensíveis, 26,7\% com sensibilidade intermediária e nenhuma cepa resistente (COUTINHO, 1997). O itraconazol também tem sido utilizado com sucesso em cães com rinite micótica (MUIR $\boldsymbol{e t}$ al., 1998; SMITH $\boldsymbol{e t}$ al., 1998) e, em micoses sistêmicas, como blastomicose, na dose de $5 \mathrm{mg} / \mathrm{kg} / \mathrm{dia}$ (LEGENDRE et al., 1996). O uso deste medicamento em cães pode levar a erupções cutâneas (PLOTNICK $\boldsymbol{e t}$ al., 1997) e, em dosagens elevadas, causar anorexia e aumento da concentração plasmática das enzimas fosfatase alcalina e aminotransferase (LEGENDRE et al., 1996). MANCIANTI et al. (1998) sugerem a dosagem de 1,5 a $3,0 \mathrm{mg} / \mathrm{kg}$ de intraconazol para gatos com dermatofitose por Microsporum canis, relatando que dosagens maiores podem causar vômitos e anorexias. JAHAM et al. (2000) indicam para micoses superficiais de cães até $5 \mathrm{mg}$ de itraconazol $/ \mathrm{kg} / \mathrm{dia}$ e $1,5-3 \mathrm{mg} / \mathrm{kg} / \mathrm{dia}$ para gatos, enquanto as micoses subcutâneas e profundas de caninos e felinos seriam tratadas com 10$20 \mathrm{mg} / \mathrm{kg} / \mathrm{dia}$ (Tabela 2).

O fluconazol é um antifúngico triazólico, que age na inibição da síntese de esteróide fúngico e praticamente não altera a síntese do ergosterol dos mamíferos, sendo menos tóxico e melhor absorvido que os outros azóis. Este antifúngico tem sido utilizado rotineiramente em pacientes humanos com micoses superfíciais e sistêmicas, levando a problemas de resistência de cepas fúngicas, principalmente em indivíduos imunossuprimidos, portadores de candidose principalmente por Candida krusei, Candida glabrata e Candida tropicalis (FAVEL et al., 1995; WALSH, et al., 1997; VAN DEN BOSSCHE, 1997). Testes de sensibilidade com Malassezia pachydermatis apresentou 66,7\% das amostras sensíveis, 23,3\% intermediárias e $10 \%$ resistentes (COUTINHO, 1997). Em estudo in vitro com 114 cepas de Cryptococcus neoformans, isolados de pacientes humanos e animais (cães e gatos) GARGANI \& PINI (1992) demonstraram um grande intervalo de variação da CMI (0,39$25,0 \mu \mathrm{m} / \mathrm{m} \ell$ ). Na literatura consultada foram poucas as referências do emprego do fluconazol em casos clínicos de animais, como KERWIN et al. (1998) que trataram com sucesso um Doberman Pinscher com criptococose associando 5,5mg de fluconazol $/ \mathrm{kg}$ de peso corpóreo e procedimento cirúrgico. JAHAM et al. (2000) recomendam dosagens de $2,5-5 \mathrm{mg} / \mathrm{kg} /$ dia para infecções fúngicas de cães e gatos (Tabela 2).

O voriconazol é um novo antifungico triazólico com potente ação e largo espectro de atividade in vitro, atua como outros antifúngicos azólicos inibindo a enzima 14 alfa-desmetilase, dependente do citocromo P-450, essencial para a biossíntese de ergosterol. O voriconazol é ativo in vitro frente a uma série de infecções fúngicas em modelos animais, incluindo infecções sistêmicas com Aspergillus sp, Candida sp, menos sensíveis a outros azólicos, e $\boldsymbol{C}$. neoformans (KAPPE, 1999; MANAVATHU et al., 2000). Estudos do efeito in vitro do voriconazol, sobre alguns fungos filamentosos, demonstrou ser ativo contra Aspergillus fumigatus, A. flavus, Fusarium oxysporum, F. solani, Fusarium spp, Pseudallescheria boydii, Rhizopus spp, A. niger, A. terreus, Acremonium spp, Paecilomyces spp e Trichoderma spp (MARCO et al., 1998). Os mesmos autores compararam o efeito do voriconazol com o do itraconazol, sendo que o primeiro foi mais ativo sobre todos os isolados com exceção do Rhizopus spp e comparando com o efeito da anfotericina B, o voriconazol foi mais efetivo contra A. fumigatus, A. flavus e $\boldsymbol{P}$. boydii e foi mais ativo que a flucitosina em todas as espécies testadas. Estudo de aspergilose invasiva em pacientes humanos imunocomprometidos, transplantados e em casos de falha de outros antifúngicos, demonstrou que o voriconazol foi efetivo contra o Aspergillus spp e bem tolerado pelos pacientes (DENNING $\boldsymbol{e t}$ 
al., 2000). BRUMMER $\boldsymbol{e}$ t al. (1998) estudaram a concentração e o tempo de ação do voriconazol e fluconazol frente ao Cryptococcus neoformans var. neoformans "ïn vitro", demonstrando que $0,2 \mu \mathrm{g} / \mathrm{m} \ell$ de voriconazol causa efeito similar na diminuição da viabilidade das células do microrganismo que $2,0 \mu \mathrm{g} / \mathrm{m} \ell$ de fluconazol, embora o número médio de células após 72 horas foram significantemente menores para o voriconazol (9) do que do fluconazol (25).

O posaconazol é um novo triazol, em estudo, que apresenta potente inibição do citocromo P-450, inibindo a síntese do ergosterol da parede celular do fungo, tendo um potente espectro de ação atuando contra Candida spp, Cryptococcus neoformans, Blatomyces dermatitidis, Histoplasma capsulatum, Sporothrix schenckii, Coccidioides immitis e Aspergillus spp (KONTOYIANNIS, 2000). O posaconazole apresenta potente atividade fungicida contra Aspergillus spp, comparado ao intraconazol ou anfotericina B ou voriconazole (KONTOYIANNIS, 2000; MANAVATHU et al., 2000).

A terbinafina é um antifúngico químico, do grupo das alilaminas, que inibe a ação da esqualeno epoxidase, enzima importante para a formação do ergosterol na membrana fúngica, resultando em morte celular. O príncipio ativo tem ação fungicida em dermatófitos (Tabela 1) e no gênero Candida apresenta efeito fungicida ou fungistático dependendo da espécie considerada. A droga não interfere no metabolismo de hormônios ou outros mecanismos, liga-se fortemente as proteínas plasmáticas, difundindo-se rapidamente através da derme e concentra-se no estrato córneo lipofílico. Em humanos, menos de 5\% da dose são absorvidos após aplicação tópica e a biotransformação resulta em metabólitos sem atividade fúngica excretados pela urina, com meia-vida de eliminação de 17 horas. Os efeitos colaterais são leves ou moderados e temporários e os sintomas mais freqüentes são gastrointestinais ou reações cutâneas sem gravidade (RICHARDSON \& WARNOCK, 1993). A droga recentemente está sendo estudada em animais de companhia e sugere-se para gatos dosagem de até $20 \mathrm{mg} / \mathrm{kg}$ a cada 24 ou 48 horas (JAHAM et al., 2000) (Tabela 2).

A flucitosina ou 5-fluorcitosina é convertida em 5-fluorouracil na célula fúngica, pela enzima citosina desaminase, que é metabolizado em ácido 5-fluorodesoxiuridílico, o qual inibe a timidilato sintetase. O 5 fluorouracil é incorporado ao RNA em substituição ao uracil, alterando a síntese protéica na célula fúngica. As células dos mamíferos não convertem grandes quantidades de flucitosina em 5-fluorouracil, o que é determinante para a ação seletiva desses compostos. A droga é rapidamente absorvida pelo trato gastrointestinal, distribuindo-se amplamente pelo corpo, atingindo concentrações elevadas no líquido cefalorraquidiano. Os efeitos colaterais podem ser anemia, leucopenia e trombocitopenia, náuseas, vômitos, diarréia e enterocolite grave e hepatomegalia (SANDE \& MANDELL, 1987; RICHARDSON \& WARNOCK, 1993). A flucitosina atua contra Cryptococcus neoformans, Candida sp, Cladosporium sp, Phialophora, não tendo efeito sobre Sporothrix schenckii, Blastomyces dermatitidis, Histoplasma capsulatum, Coccidioides immitis, Absídia e Rhizopus (SANDE \& MANDELL, 1987; LACAZ \& NEGRO, 1991; RICHARDSON \& WARNOCK, 1993). Para os animais está indicado em dosagens de 125 a 250mg de flucitosina $/ \mathrm{kg}$ de peso/dia, principalmente em gatos com criptococose, utilizando-a associada a anfotericina B (PRESCOTT \& BAGGOT, 1991) (Tabela 1 e 2). A manifestação de alterações cutâneas como: despigmentação, exsudação, ulceração e formação de crostas, foi descrita em cães com criptococose ou aspergilose tratados com associação flucitosina e anfotericina B e/ou triazoles, sendo que, com a suspensão da flucitosina e a manutenção do restante dos medicamentos cessaram as alterações (MALIK $\boldsymbol{e t}$ al., 1996b)

\section{ANTIBIÓTICOS}

Os antibióticos poliênicos possuem um grande anel lactônico macrocíclico e semelhante aos macrolídios antibacterianos como a eritromicina, daí serem chamados macrolídios poliênicos, sendo a estrutura ativa o anel macrolídio com suas partes rígida lipofílica e flexível hidrofílica (SANDE \& MANDELL, 1987). A anfotericina B é um antibiótico poliênico derivado do Streptomyces nodosus, cujo mecanismo de ação, se baseia na ligação com os esteróis da membrana celular, provocando uma alteração funcional com saída de metabólitos essenciais, nucleotídeos e proteínas, levando à morte celular (SANDE \& MANDELL, 1987; RICHARDSON \& WARNOCK, 1993).

MALECAZE $\boldsymbol{e t}$ al. (1992) recomenda a anfotericina B local no tratamento das queratomicoses, pelo sua rápida penetração no tecido e diminuição do efeito tóxico. No tratamento de micoses sistêmicas, em cães, com anfotericina B deve ser avaliado o estado geral do animal, recomendando para animais em bom estado geral $0,5 \mathrm{mg} / \mathrm{kg}$ de peso via endovenosa, três vezes por semana, com controle do nitrogênio sangüíneo, em função da toxicidade renal. Em cães debilitados utilizar dose inicial de $0,2 \mathrm{mg} / \mathrm{kg}$ de peso via 
endovenosa, aumentando diariamente a dose em $0,1 \mathrm{mg} / \mathrm{kg}$ de peso, até atingir $0,6 \mathrm{mg} / \mathrm{kg}$, sendo a dose a ser mantida, administrada em dias alternados (PRESCOTT \& BAGGOT, 1991) (Tabela 1 e 2). A maioria dos cães submetidos a anfotericina B manifesta febre leve a moderada, perdurando até 36 horas após a administração. A toxicidade renal é um efeito colateral importante em cães, não sendo rapidamente reversível pela suspensão da droga como em humanos (ARONSON \& AUCOIN, 1992; MONTEIRO, et al., 1993; RADALL et al., 1996). Novas formulações de anfotericina B com lipídios para infusão intravenosa, consideradas menos toxicas aos mamíferos, estão em desenvolvimento (ALBERT et al., 1995; SPERRY et al., 1998). Estudo em cães, com blastomicose, tratados com complexo lipídico de anfotericina B demonstraram o efeito curativo do tratamento e ausência de efeitos nefrotoxicos (KRAWIEC et al., 1996).

No tratamento da criptococose felina, a anfotericina B é recomendada nas doses de 0,1 a $0,5 \mathrm{mg} / \mathrm{kg}$ de peso, a cada dois ou três dias (BARRET \& SCOTT, 1975; PALUMBO \& PERRY, 1975). A anfotericina B foi utilizada por via subcutânea em seis casos de criptococose em gatos e cães, com dose entre 0,5 a $0,8 \mathrm{mg} / \mathrm{kg}$ em solução salina $(0,45 \%)$ e dextrose $(2,5 \%)$, aplicada duas a três vezes por semana durante vários meses, sendo efetiva e bem tolerada pelos animais, com alguns casos de irritação local e de azotemia (MALIK et al., 1996a). Nos eqüinos, em casos de zigomicoses, a anfotericina $\mathrm{B}$ é recomendada em doses entre 0,5 e $1,5 \mathrm{mg} / \mathrm{kg}$ de peso diluído em 1 litro de solução de dextrose a 5\%, para aplicação endovenosa (McMULAN et al., 1977) (Tabela 2).

A anfotericina B aumenta a atividade da 5-fluorocitosina porque facilita a sua entrada através da membrana citoplasmática. Em estudos iniciais de associações dos azóis com a anfotericina $\mathrm{B}$, foi descrita a freqüência do efeito antagônico da associação da anfotericina B com o cetoconazol (SANDE \& MANDELL, 1987), entretanto em estudos recentes de interações medicamentosas, incluindo os triazóis, frente a Candida $s p$, Cryptococcus neoformans, Aspergillus sp, Blastomyces dermatitidis e Histoplasma capsulatum os resultados variaram entre indiferença, sinergismo e o antagonismo (HENNEQUIN $\boldsymbol{e t}$ al., 1992; KOWALEWICH $\boldsymbol{e}$ t al. 1993; BLOOM et al., 1996; ARCENEAUX et al.; 1998; POLAK, 1999).

A nistatina, isolada do Streptomyces noursei, pertence ao grupo dos antibióticos poliênicos. É levemente hidrosolúvel e atua lesando a membrana citoplasmática dos fungos sensíveis e sua ação pode ser tanto fungicida como fungistática, estando indicada como terapia tópica em micoses superficiais, de pele e mucosa, principalmente na candidose (Tabela 1), sendo extremamente tóxica para uso parenteral. Nas doses terapêuticas, por via oral, a absorção pelo trato gastrointestinal é praticamente inexistente (SANDE \& MANDELL, 1987). Em pequenos animais, cães e gatos, é utilizada no tratamento de otomicose por leveduras e em éguas está recomendada no tratamento de metrite por Candida sp (PRESCOTT \& BAGGOT, 1991). Atualmente uma nova formulação da nistatina, nistatina lipossomal, está sendo utilizada, com menos efeitos tóxicos que a fórmula original, causando menos hemólise como efeito colateral. A nistatina lipossomal tem largo espectro de ação atuando contra Allescheria sp, Alternaria sp, Aspergillus flavus, Aspergillus fumigatus, Aspergillus niger, várias espécies de Candida, Cladosporium, Cryptococcus neoformans, Mucor, Penicilium, Rhizopus, Rhodotorulla, Sporothrix sp, Trichosporon sp, entre outros, mas não foi ativa contra algumas cepas de Fusarium testadas. Contra leveduras resistentes in vitro a outros antifúngicos como fluconazol, itraconazol e anfotericina B ela foi efetiva (HERBRECHT, 2000).

A natamicina, também um antibiótico poliênico, é utilizada em aplicações tópicas para combater mastites por leveduras e nas queratites micóticas (Tabela 1), não sendo indicada nas infecções profundas do olho, porque sua absorção é escassa (PRESCOTT \& BAGGOT, 1991).

A griseofulvina é um antibiótico que foi isolado pela primeira vez do Penicillium griseofulvum e, devido ao fato de ser ineficaz contra bactérias, não foi utilizada. Após alguns anos, foi encontrada uma substância no Penicilium janczewski que produzia contração e desnaturação das hifas fúngicas, denominada inicialmente de fator de enrugamento e posteriormente descobriram tratarse da griseofulvina. O princípio ativo tem efeito fungistático para dermatófitos (Tabela 1), e não exerce efeitos sobre outros fungos (SANDE \& MANDELL, 1987; LACAZ \& NEGRO, 1991; MORIELLO \& DEBOER, 1995). A griseofulvina é metabolizada mais rapidamente em cães do que em seres humanos, e, por essa razão, deve ser utilizada para estes animais dosagens mais elevadas, muito embora o mecanismo de eliminação da droga não tenha sido ainda completamente descrito no cão ou no gato. Nesses animais pode ser utilizada dosagens iniciais de $50 \mathrm{mg}$ de griseofulvina $/ \mathrm{kg} / \mathrm{dia}$, reduzindo para $25 \mathrm{mg} / \mathrm{kg} /$ dia conforme evolução do tratamento, durante 3 a 6 semanas no caso de dermatofitose de pele e de 6 a 12 meses nas onicomicoses 
(PRESCOTT \& BAGGOT, 1991) (Tabela 2).A toxicidade deste composto, em cães, é basicamente através de perturbações gastrointestinais. A droga é teratogênica, não sendo recomendável a animais em reprodução. Em gatos, particularmente em filhotes, a griseofulvina está relacionada à causa de leucopenia, anemia, letargia, anorexia, ataxia e depressão (PRESCOTT \& BAGGOT, 1991; ARONSON \& AUCOIN, 1992). Griseofulvina foi administrada a uma égua com dermatofitose, que estava no segundo mês de gestação e aos 331 dias ocorreu o nascimento do feto com alterações teratogênicas (SCHUTTE \& VAN DEN INGH, 1997).

Pneumocandinas e echinocandinas são uma nova classe de antifúngicos lipopeptídios, de uso sistêmico, em estudo, que atuam inibindo a 1,3beta-D-glucan sintetase. O cilofungin foi o primeiro destas drogas em estudo clínico e apresentou bom resultado frente a cepas resistentes a outros antifúngicos, mas por ser solúvel em polietileno glicol mostrou nefrotoxicidade. Atualmente as pesquisas estão sendo desenvolvidas no Capsofungin (MK-0991) que é solúvel em água, sendo efetivo em casos de candidoses, aspergilose e cryptococose, embora nesta última com doses mais elevadas, não tendo demonstrado nefrotoxicidade, apenas reação no local da infusão (GRAYBILL, 2000). FK463 é solúvel em água, atua da mesma maneira que os anteriores e demonstra significante inibição do crescimento da $\boldsymbol{C}$. albicans e A. fumigatus em baixas concentrações, sendo também ativo contra outras espécies de Candida, Aspergillus e outros fungos (GROLL \& WALSH, 2000).

\section{CONCLUSÕES}

Segundo a revisão, conclui-se que o crescente avanço das Ciências Micológicas e conseqüentemente o aumento da casuística de micoses animais, se torna necessário à pesquisa e ao desenvolvimento de princípios ativos, seus efeitos curativos e colaterais para o organismo dos animais. Pouco se conhece, até o momento, do uso de antifúngicos na clínica veterinária diária e a terapêutica continua sendo ancorada em produtos de uso humano, demonstrando a necessidade de estudos das propriedades farmacodinâmicas e farmacocinéticas nas várias espécies animais.

Esta revisão tenta de uma forma resumida trazer à luz do conhecimento da clínica diária o uso de alguns princípios de antifúngicos e seus efeitos adversos, possibilitando a utilização em formulações genéricas quando na falta da formulação comercial para animais.
ALBERT, M.M.; STAHL-CARROL, T.L.; LUTHER, M.F., et al . Comparison of lipossomal amphotericin B to amphotericin B for treatment of murine cryptococcal meningitis. J. Mycol Med, v.5 p.1-6, 1995

ALVES, S.H.; LOPES, J.O.; CURY, A.E. Teste de suscetibilidade aos antifúngicos: por que, quando e como realizar. Disponível on line na Internet em 05 de abril de 1999. http://www.newslab.com.br/antifung.htm

ANDREW, S.E.; BROOKS, D.E.; SMITH, P.J., et al. Equine ulcerative keratomycosis: visual outcome and ocular survival in 39 cases (1987-1996). Equine Vet J, v.30, n.2, p.109-116, 1998.

ARCENEAUX, K.A.; TABOADA, J.; HOSGOOD, G. Blastomycosis in dogs. 115 cases (1980-1995). J Am Vet Med Assoc, v.213, n.5, p.658-664, 1998.

ARENAS, R. Micologia medica ilustrada. México : Nueva editorial interamericana, 1993. Cap.34.: Antimicoticos; p.359-376.

ARONSON, A.; AUCOIN, D.P. Medicamentos antimicrobianos In: ETTINGER, S.J. Tratado de medicina interna veterinária. São Paulo : Manole, 1992. Cap.51. p.402-431.

BARRET, R.E.; SCOTT, D.W. Treatment of feline cryptococosis: literature review and case report. J Am Anim Hosp Assoc, v.11, p.511, 1975.

BLOOD, D.C.; RADOSTITS, O.M. Clínica veterinária. 7 ed., Rio de Janeiro, RJ : . Guanabara, 1991. Cap.24: Doenças causadas por fungos. p.814-822.

BLOOM, J.D.; HARMOR, R.E.; GERDING, P.A. Ocular blastomycosis in dogs: 73 cases, 108 eyes (1985-1993). J Am Vet Med Assoc, v.209, n.7, p.1271-1274, 1996.

BOND, R.; ROSE, J.F.; ELLIS, J.W., et al. Comparison of two shampoos for treatment of Malassezia pachydermatis associated seborrhoeic dermatitis in basset hound. J Small Anim Pract, v.36, n.3, p.99-104, 1995.

BRAY, J.P.; WHITE, R.A.; LASCELLES, B.D. Treatment of canine nasal aspergillosis with a new non-invasive technique Failure with eniconazole. J Small Anim Pract, v.39, n.5, p.223-226, 1998.

BRUMMER, E.; KAMEI, K.; MIYAJI, M. Damage to yeast cells of Cryptococcus neoformans by voriconazole and fluconazole: a culture and microscopic study. Med Mycol, v.36, n.4, p.227-233, 1998.

CAULKETT, N.; LEW, L.; FRIES, C. Upper-airway obstruction and prolonged recovery from anesthesia following intranasal clotrimazole administration. J Am Anim Hosp Assoc, v.33, n.3, p.264-267, 1997.

CLERCX, C. Treatment of canine nasal aspergillosis. Disponível on line na Internet em 16 de dezembro de 2000. http://www.aspergillus.

COUTINHO, S.D.A. Malassezia pachydermatis: caracterização fenotípica de amostras isoladas de pelame e meato acústico externo de cães. São Paulo- SP, 1993. 108p. Tese (Doutorado em Ciências) - Instituto de Ciências Biomédicas da Universidade de São Paulo, 1997. 
DAVIS, E.W.; LEGENDRE, A.M. Seccessful tratment of guttural pouch mycosis with itraconazole and topical enilconazole in horse. J Vet Intern Med, v.8, n.4, p.304$305,1994$.

DENNING, D.W.; FAVERO, A.; GLUCKMAN, E., et al. The efficacy and tolerability of UK 109,496 (voriconazole) in the treatment of invasive aspergilosis (IA). Disponível on line na Internet em 16 de dezembro de 2000 http://www.aspergillus.

FAVEL, A.; LIEBERMANN, M.; MICHEL-NGUYEN, A., et al. Fluconazole susceptibility testing of Candida species: a compartive study of RPMI, high resolution and casitone media. J Mycol Med, v.5, p.7-12, 1995.

GABAL, M.A. Antifungal activity of ketoconazole with emphasis on zoophilic fungal pathogens. Am J Vet Res, v.47, p.229-1234, 1986.

GARGANI, G.; PINI, G. Caractéres mycologiques et sensibilité aux antifongiques des souches de Cryptococcus neoformans isolées de patients atteints du sida ou d'autres provenances. J Mycol Med, v.2, p.140-143, 1992.

GRAYBILL, J. New Drugs. Meck-0991/Capsofungin Disponível on line na Internet em 16 de dezembro de 2000. http://www.aspergillus.

GROLL, A.H.; WALSH, T.J. New Drugs. FK463, A novel Echinocandin-like antifungal lipopeptide.Disponível on line na Internet em 16 de dezembro de 2000 http://www.aspergillus

HELL, R.C.; BROGDEN, R.N.; SPEIGHT, T.M., et al. Econazole: a review of its antifungal activity and therapeutic efficacy. Drugs, v.16, n.3, p.177-201, 1978.

HENNEQUIN, C.; IMPROVIST, L; BOIRON, P., et al. Étude in vitro des association amphotéricine $\mathrm{B}$ - azolés vis-à-vis de Candida albicans et de Candida tropicalis. J Mycol Med, v.2:68-72, 1992.

HERBRECHT, R. New drugs. Lipossomal nystatin (Nyotran®). Disponível on line na Internet em 16 de dezembro de 2000. http://www.aspergillus.

JAHAM, C.; PARADIS, M.; PAPICH, M.G. Antifungal dermatologic agents: azoles and allylamines. Small Anim/Exotics, v.22, n.6, p.548-558, 2000.

KAPPE, R. Antifungal activity of the new azole UK-109,496 (voriconazole). Mycoses, v.42, n.2, p.83-86, 1999.

KERWIN, S.C.; MCCARTHY, R.J.; VANSTEENHOUSE, J.L., et al. Cervical spinal cord compression causaded by cryptococcosis in a dog: successful treatment with surgery and fluconazole. J Am Hosp Assoc, v.34, n.6, p.523-526, 1998.

KONTOYIANNIS, D.P. New Drugs. Posaconazole. Disponível on line na Internet http://www.aspergillus. 16/12/2000.

KOWALEWICH, N.; HAWKINS, E.C.; SKOWRONEK, A.J., et al. Identificationa of Histoplasma capsulatum organisms in the pleural and peritoneal effusions of a dog. J Am Vet Med Assoc, v.202, n.3, p.423-426, 1993.
KRAWIEC, D.R.; MCKIERMAN, B.C.; TWARDOCK, A.R., $\boldsymbol{e} t$ al. Use of na anfotericin B lipid complex for treatment of blastomycosis in dogs. J Am Vet Med Assoc, v. 209, n.12, p.2073-2075, 1996.

LACAZ, C.S.; NEGRO, G. Drogas antifúngicas. Terapêutica das micoses. In: LACAZ, C.S.; PORTO, E.; MARTINS, J.E.C. Micologia médica fungos, actinomicetos e algas de interesse médico. São Paulo : Savier, 1991. Cap.38. p.616-651.

LEGENDRE, A.M.; ROHRBACH, B.W.; TOAL, R.L., et al. Treatment of blastomycosis with itraconazole in 112 dogs. J Vet Intern Med, v.10, n.6, p.365-371, 1996.

LOBELL, R.; WEINGARTEN, A.; SIMMONS, R. Um novo agente para o tratamento da otite externa canina. A Hora Vet, v.88, p.29-33, 1995.

MALECAZE, F.; LINAS, M.D.; GAZAGNE, C., $\boldsymbol{e} \boldsymbol{t} \boldsymbol{a l}$. Diffusion intraoculaire des antifongiques et conséquences thérapeutiques dans les mycoses oculaires. J Mycol Med, v.2: $73-76,1992$.

MALIK, R.; CRAIG, A.J.; WIGNEY, D.I., et al. Combination chemotherapy of canine and feline cryptococcosis using subcutaneously administered amphotericin B. Aust Vet J, v.73, n.4, p.124-128, 1996a.

MALIK, R.; MEDEIROS, C.; WIGNEY, D.I., et al. Suspected drug eruption in seven dogs during administration of flucytosine. Aust Vet J, v.74, n.40, p.285-288, 1996b.

MANAVATHU, E.K.; CUTRIGHT, J.L.; LOEBENBERG, D., et al. A comparative study of the in vitro susceptibilities of clinical and laboratory-selected resistant isolates of Aspergillus spp. to amphotericin B, itraconazole, voriconazole and posaconazole (SCH 56592). J Antimicrob Chermother, v.46, n.2, p.229-234, 2000.

MANCIANTI, F.; PEDONESE, F.; ZULLINO, C. Efficacy of oral administration of itraconazole to cats with dermatophytosis caused by Microsporum canis. J Am Vet Med Assoc, v.213, n.7, p.993-995, 1998.

MARCO, F.; PFALLER, M.A.; MESSER, S.A., et al. Antifungal activity of a new triazole, voriconazole (UK-109,496), compared with three other antifungal agents tested against clinical isolates of filamentous fungi. Med Mycol, v.36, p.433-436, 1998

MATHEWS, K.G.; DAVIDSON, A.P.; KOBLIK, P.D., et al. Comparison of topical administration of clotrimazole through surgically placed versus nonsurgically placed datheters for treatment of nasal aspergilosis i dogs: 60 cases (1990-1996). J Am Vet Med Assoc, v.213, n.4, p.501-506, 1998.

McCULLOUGH, S.M.; McKIEMAN, B.C.; GRODSKY, B.S Endoscopically placed tubes for administration of enilconazole for treatment of nasal aspergillosis in dogs. $\mathbf{J}$ Am Vet Med Assoc, v.212, n.1, p.67-79, 1998.

McCURDY, H.D.; HEPLER, D.I.; LARSON K.A. Effectiveness of a topical agent (clotrimazole) in dogs. J Am Vet Med Assoc, v.179, n.2, p.163-165, 1981.

McMULLAN, W.C.; JOYCE, J.R.; HANSELKA, D.V., et al. Amphotericin B for the treatment of localized subcutaneous phycomycosis in the horse. J Am Vet Med Assoc, v.170, n.11, p.1293-1298, 1977. 
MONTEIRO, J.L.; SEGURO, A.C.; ROCHA, A.S. Nefrotoxicidade aguda da anfotericina B no cão. Rev Hosp Clin Fac Med São Paulo, v.48, n.2, p.54-59, 1993.

MORIELLO, K.A. Ketoconazole: clinical pharmacologic and therapeutic recommendations. J Am Vet Med Assoc, v.188, p. 303,1986

MORIELLO, K.A.; DEBOER, D.J. Efficacy of griseofulvin and itraconazole in the treatment of experimentally induced dermatophytosis in cats. J Am Vet Med Assoc, v.207, n.4, p.439-444, 1995

MUIR, D.; MARTIN, P.; KENDALL, K., et al. Invasive hyphomycotic rhinitis in a cat due Metarhizium anisopliae. Med Mycol, v.36, n1, p.51-54, 1998.

NOXON, J.O.; DIGILIO, K.; SCHMID, T.D.A. Disseminated histoplasmosis in a cat: suscessful treatment with ketoconazole. J Am Vet Med Assoc, v.181, n.8, p.817-820, 1982.

PALUMBO, N.E.; PERRI, S. Anphotericin B therapy in two cases of feline cryptococosis. Vet Med Small Anim Clin, v.70, n.5, p.553, 1975 .

PLOTNICK, A.N.; BOSHOVEN, E.W.; ROSYCHUK, R.A. Primary cutaneous coccidioidomycosis and subsequent drug eruption to itraconazole in a dog. J Am Anim Hosp Assoc, v.33, n.2, p.139-143, 1997.

POLAK, A. The past, present and future of antimycotic combination therapy. Mycoses, v.42, p.335-370, 1999.

PRESCOTT, J.F.; BAGGOT, J.D. Terapêutica antimicrobiana veterinária. Zaragoza : Acribia, 1991. Cap.15: Quimioterapia antifúngica: p.309-330.

RANDALL， S.R.; ADAMS， L.G.; WHITE, M.R., et al. Nephrotoxicity of amphotericin B administered to dogs in a fat emulsion versus five percent dextrose solution. Am J Vet Res, v.57, n.7, p.1054-1058, 1996.

RICHARDSON, M.D.; WARNOCK, D.W. Fungal infection Diagnosis and management. London : Blackwell, 1993. Cap.3: Antifungal drugs: 17-43.

SANDE, M.A.; MANDELL, G.L. Drogas antimicrobianas Drogas antimicóticas e antivirais. In: GOODMAN, L.; GILMAN, A.G. As bases farmacológicas da terapêutica. Rio de Janeiro : Guanabara, 1987. Cap.54. p.799-807.

SAWYER, P.R.; BROGDEN, R.N.; PINDER, R.M., et al. Clotrimazole:a review of its antifungal activity and therapeutic efficacy. Drugs, v.9, n.6, p.424-447, 1975a.

SAWYER, P.R.; BROGDEN, R.N.; PINDER, R.M., et al. Miconazole:a review of its antifungal activity and therapeutic efficacy. Drugs, v.9, n.6, p.406-423, 1975b.

SCHUTTE, J.G.; VAN DEN INGH, T.S. Microphthalmia, brachygnathia superior, and palatocheiloschisis in a foal associated with griseofulvin administration to the mare during esrly pregnancy. Vet Q, v.19, n.2, p.58-60, 1997.

SMITH, S.A.; ANDEWS, G.; BILLER, D.S. Management of nasal aspergilosis in a dog with a single, noninvasive intranasal infusion of clotrimazole. J Am Anim Hosp Assoc, v.34, n.6, p.487-492, 1998.

SPERRY, P.J.; SUA, D.J.; WETZEL, S.A., et al. Antimicrobial activity of AmBisome and non-liposomal amphotericin B following uptake of Candida glabrata by murine epidermal Langerhans cells. Med Mycol, v.36, p.135-141, 1998.

UCHIDA, Y.; NAKADE, T.; KITAZAWA, K. In vitro activity of five antifungal agents against Malassezia pachydermatis. Nippon Juigaku zassihi, v.52, n.4, p.851-853, 1990.

VAN DEN BOSSCHE, H. Mechanisms of antifungi resistance. Rev Iberoam Mico, v.14, n.2, p.44-49,1997.

VAN NIEUWSTADT, R.A.; KALSBEEK, H.C. Air sac mycosis: topical treatment using enilconazole administered via indwelling catheter. Tijdschr diergeneeskd, v.119, n.1, p.3-5, 1994

WALSH, T.J.; KASAI, M.; FRANCESCONI, A., et al. New wvidence that Candida albicans possesses additional ATPbinding cassete MDR-like genes: implications for antifungal azole resistance. J Med Vet Mycol, v.35, n.2, p.133$137,1997$.

WEIGHT, U.; AHLERS, D. Zur atilogie, symptomatologie und therapie der hefemastitis beim rind. DTW, v.89, n.6, p.234$238,1982$.

WHITE-WEITHERS, N.; MEDLEAU, L. Evaluation of topical therapies for treatment of dermatophyte-infected hairs from dogs and cats. J Anim Hosp Assoc, v.31, n.3, p.250-253, 1995

WILLARD, M.D. NACHREINER, R., MCDONALD, R., et al., Ketoconazole-induced changes in selected canine hormone concentrations. Am j Vet Res, v.47, n.2, p.2504-2509, 1986.

WOLF, A.M.; GREGORY, C.T. Moléstias micóticas profundas. In: ETTINGER, S.J. Tratado de medicina interna veterinária. São Paulo : Manole, 1992. Cap.49. p.357-369. 